\title{
Interionic Force Model for Pentahalide Molecules and Higher Niobium-Based Halide Clusters
}

\author{
Z. Çiçek Önem, Z. Akdeniz, and M. P. Tosi ${ }^{\mathrm{a}}$ \\ Department of Physics, University of Istanbul, Istanbul, Turkey \\ and Abdus Salam ICTP, Trieste, Italy \\ ${ }^{a}$ INFM and Classe di Scienze, Scuola Normale Superiore, I-56126 Pisa, Italy \\ Reprint requests to Prof. M. P. T.; Fax: +39-050-563513; E-mail: tosim@sns.it
}

Z. Naturforsch. 57 a, 943-947 (2002); received August 23, 2002

\begin{abstract}
Molecular bound states tend to become progressively more stable in the melts of polyvalent metal halides as the nominal valence of the metal increases. We examine in this work the case of pentavalent metal halides. First we propose a simple ionic model for the binding in several pentahalide clusters: the chlorides of $\mathrm{Nb}$, Ta, $\mathrm{Sb}$, and $\mathrm{Mo}$ and the bromides of $\mathrm{Nb}$ and $\mathrm{Ta}$. The molecular monomers of these compounds have a $\mathrm{D}_{3 \mathrm{~h}}$ trigonal-bipyramidal structure in the ground state, and we make use of data on equatorial bond lengths and breathing mode frequencies in the vapour to determine the main force-law parameters of the metal ion. We also find that the $\mathrm{C}_{\mathrm{V}}$ square-pyramidal structure is mechanically unstable against transformation into the $\mathrm{B}_{\mathrm{h}}$ shape. We then consider higher molecular clusters, $i$. e. the dimers of $\mathrm{Nb}$ pentahalides and the bound states formed by $\mathrm{NbCl}_{5}$ with the chlorides of $\mathrm{Cs}, \mathrm{Al}, \mathrm{Ga}$, and $\mathrm{Sb}$. We propose structural models for all these stable clusters and compare their calculated vibrational frequencies with the available data from vibrational spectroscopy of mixed melts.
\end{abstract}

Key words: Ionic Clusters; Vapour Complexes; Molten Salts.

\section{Introduction}

Charge compensation within well defined molecular units becomes frequent in the condensed phases of metal halides if the nominal valence of the metal increases [1]. In comparison with more conventional molten salts such as the alkali or alkaline-earth halides, the liquid phase is characterized in these molecular systems by a relatively low freezing point and by high fluidity and very low electrical conductivity. Structural characterizations through diffraction experiments or simulation studies based on interionicforce models have provided direct evidence for such units in a number of melts. Well known examples among trihalides are $\mathrm{AlBr}_{3}$ [2] and $\mathrm{SbCl}_{3}$ [3], the basic molecular units being the dimer in the former case and the monomer in the latter. These units are, of course, strongly correlated with each other in the dense melt. Molecular-liquid behaviour is similarly well established among tetrahalides for $\mathrm{GeBr}_{4}[4]$ and $\mathrm{ZrCl}_{4}$ [5]. Typical molten-salt behaviour is restored upon mixing with alkali halides, which through halogen donation to the polyvalent metal element gives rise to a liquid structure formed by complex anions and alkali counterions.

Following earlier work on the determination of interionic-force models for molecular bound states in the tetrahalides of $\mathrm{Zr}$, Th, and actinide elements [6], we examine in the present work the case of pentahalides. For the monomeric molecules of the halides of such elements as $\mathrm{Nb}$ or $\mathrm{Ta}$, the possibility exists of either a $\mathrm{D}_{3 \mathrm{~h}}$ trigonal bipyramidal shape or a $\mathrm{C}_{4 \mathrm{v}}$ tetragonal pyramidal structure [7]. The chlorides and bromides of both $\mathrm{Nb}$ and Ta crystallize into structures whose basic constituents are molecular dimers [8]. Coexistence of monomers and dimers in the melt has been reported from vibrational spectroscopy for $\mathrm{NbCl}_{5}$ and $\mathrm{SbCl}_{5}$ [9].

The paper is organized as follows. In Sect. 2 we propose a simple ionic model for a number of pentahalides for which the necessary essential data are available on the monomer in the vapour phase. These are the pentachlorides of $\mathrm{Nb}, \mathrm{Ta}, \mathrm{Sb}$, and $\mathrm{Mo}$ and the pentabromides of $\mathrm{Nb}$ and $\mathrm{Ta}$. Although $\mathrm{MoCl}_{5}$ may undergo a dynamic Jahn-Teller distortion into a $\mathrm{C}_{2 \mathrm{v}}$ structure, the available evidence is that the Jahn-Teller 
Table 1 . Effective valence $z_{\mathrm{M}}$ and ionic radius $R_{\mathrm{M}}$ of pentavalent metal ions in chloride and bromide monomers. The calculated ratio $r_{\mathrm{a}} / r_{\mathrm{e}}$ of bond lengths is also shown.

\begin{tabular}{lllllll}
\hline & $\mathrm{NbCl}_{5}$ & $\mathrm{TaCl}_{5}$ & $\mathrm{SbCl}_{5}$ & $\mathrm{MoCl}_{5}$ & $\mathrm{NbBr}_{5}$ & $\mathrm{TaBr}_{5}$ \\
\hline$z_{\mathrm{M}}$ & 3.80 & 3.98 & 3.39 & 3.75 & 3.82 & 3.85 \\
$R_{\mathrm{M}}(\AA)$ & 1.17 & 1.19 & 1.10 & 1.16 & 1.18 & 1.15 \\
$r_{\mathrm{a}} / r_{\mathrm{e}}$ & 1.009 & 1.009 & 1.011 & 1.010 & 1.009 & 1.009 \\
\hline
\end{tabular}

stabilization is very weak [10], therefore its effect is not included in our study. Section 3 presents applications of the above results to the evaluation of higher complexes for the case of the $\mathrm{Nb}$ compounds: we study the stability of the dimers of $\mathrm{NbCl}_{5}$ and $\mathrm{NbBr}_{5}$ and of the $\mathrm{NbSbCl}_{10}$ complex, as well as the consequences of chlorination through addition of $\mathrm{CsCl}$, $\mathrm{AlCl}_{3}$, and $\mathrm{GaCl}_{3}$ to $\mathrm{NbCl}_{5}$. Finally, Sect. 4 gives a brief summary of our results and some concluding remarks.

\section{Interionic Force Model}

The model that we adopt in this work for pentahalide compounds was earlier developed for trihalides [11] and tetrahalides [6]. It is based on the Born model for cohesion in ionic compounds and on the shell model for lattice vibrations. Electron-shell deformability is taken into account through (i) effective valences $z_{i}$ subject to overall charge neutrality, and (ii) electric and overlap polarizabilities for the halogen ions. Van der Waals dipole-dipole interactions are included between the halogens. Overlap repulsions $\Phi_{i j}\left(r_{i j}\right)$ of exponential form between pairs of ions at separation $r_{i j}$ involve ionic radii $R_{i}$ and stiffness parameters $\rho_{i}$, according to

$$
\Phi_{i j}\left(r_{i j}\right)=f\left(\rho_{i}+\rho_{j}\right) \exp \left[\frac{R_{i}+R_{j}-r_{i j}}{\rho_{i}+\rho_{j}}\right],
$$

where $f=0.05 e^{2} / \AA^{2}$ is a constant fixing the energy scale. The reader is referred to our earlier work [11] for details on the other contributions to the potential energy of a cluster as a function of the interionic distances and of the electric dipole moments, as well as for a description of the numerical method used to calculate equilibrium structures and vibrational frequencies.

In determining the parameters of the model for pentahalides, we have made use of the simplifying features that have previously been established in similar
Table 2. Vibrational frequencies of pentavalent-halide monomers (in $\mathrm{cm}^{-1}$ ), from the ionic model (IM) and from data on the vapour phase $(\mathrm{V})$. The frequencies of the $\nu_{1}$ breathing mode have been fitted to experiment.

\begin{tabular}{lccccccccr}
\hline & & $\nu_{1}$ & $\nu_{2}$ & $\nu_{3}$ & $\nu_{4}$ & $\nu_{5}$ & $\nu_{6}$ & $\nu_{7}$ & $\nu_{8}$ \\
\hline $\mathrm{NbCl}_{5}$ & IM & 394 & 314 & 429 & 168 & 430 & 164 & 55 & 153 \\
& V & 394 & 317 & 396 & 126 & 444 & 159 & 99 & 148 \\
$\mathrm{TaCl}_{5}$ & $\mathrm{IM}$ & 406 & 333 & 413 & 160 & 411 & 157 & 57 & 155 \\
& V & 406 & 324 & - & - & - & 181 & 54 & 127 \\
$\mathrm{SbCl}_{5}$ & IM & 355 & 275 & 357 & 152 & 364 & 147 & 51 & 144 \\
& V & 355 & 309 & - & - & 400 & 173 & 58 & 120 \\
$\mathrm{MoCl}_{5}$ & IM & 390 & 310 & 420 & 166 & 422 & 163 & 54 & 152 \\
& V & 390 & 313 & - & - & 418 & 200 & 100 & 175 \\
$\mathrm{NbBr}_{5}$ & IM & 234 & 192 & 324 & 109 & 316 & 109 & 33 & 92 \\
& V & 234 & 178 & - & - & - & 119 & 67 & 101 \\
$\mathrm{TaBr}_{5}$ & IM & 240 & 195 & 274 & 106 & 273 & 104 & 34 & 95 \\
& V & 240 & 182 & - & - & - & 110 & 70 & 93 \\
\hline
\end{tabular}

studies of other ionic compounds, which are the transferability of parameters describing the halogen ions and the direct proportionality between the characteristic radius $R_{\mathrm{M}}$ and the hardness parameter $\rho_{\mathrm{M}}$ of the metal ion. Here we need to report, therefore, only on the values that we have determined for the valence $z_{\mathrm{M}}$ and the radius $R_{\mathrm{M}}$ of the pentavalent metal ions, corresponding to the choice $R_{\mathrm{M}} / \rho_{\mathrm{M}}=18.6$ and to the values of the model parameters for the chlorine and bromine ions reported in [6]. These two parameters, whose values are given in the first two rows of Table 1 , have been determined by adjusting the model to vapour-phase data on monomers in the $\mathrm{D}_{3 \mathrm{~h}}$ structure, which are (i) the equatorial metal-halogen bond length from electron diffraction data $[10,12]$, and (ii) the frequency of the molecular breathing mode [9]. The main point to be noted is that the effective valences in Table 1 are appreciably smaller than the nominal valence of the metal ions. This implies that an important contribution to the cohesion of these molecules is coming from the electronic polarization of the halogens, which within a pseudoclassical model mimics the role of quantum-chemical bonding.

The last row in Table 1 reports the calculated values of the ratio $r_{\mathrm{a}} / r_{\mathrm{e}}$ between the axial and equatorial bond lengths in the $\mathrm{D}_{3 \mathrm{~h}}$ ground state of the monomer. We find that the axial bond lengths are elongated by about $1 \%$, in agreement with the electron diffraction data [10].

The vibrational frequencies that we have calculated for each cluster are reported in Table 2 and compared with the available data from vapour-phase or matrix vibrational spectroscopy, from the review by Brooker 
and Papatheodorou [9]. The model reproduces the main trends of the data and is quantitatively quite good for the high-frequency modes.

Finally, with the same model parameters we have examined the pentahalide monomers in the $\mathrm{C}_{4 \mathrm{v}}$ tetragonal-pyramid shape. We have found that four halogens are arranged in a square forming the base of the pyramid and the metal ion is somewhat displaced out of the basal plane towards the fifth halogen at the apex of the pyramid. The cohesive energies and the bond lengths of the monomers in the $\mathrm{C}_{4 \mathrm{v}}$ structure and in the $D_{3 h}$ structure are very similar: for instance, for $\mathrm{NbCl}_{5}$ we find that the equatorial (axial) bond lengths are $2.29(2.27) \AA$ in the former structure against 2.28 (2.30) $\AA$ in the latter. However, in the $\mathrm{C}_{4 \mathrm{v}}$ configuration a vibrational mode has an imaginary frequency and drives the monomer to transform into the $D_{3 h}$ shape by a process of axial-equatorial exchange, as already discussed by Kang et al. [13].

\section{Higher Complexes}

We have used the ionic model presented in Sect. 2 to evaluate the structure, the binding energy, and the vibrational frequencies of a number of higher complexes involving the $\mathrm{Nb}$ metal ion. These are (i) the dimers of $\mathrm{NbCl}_{5}$ and $\mathrm{NbBr}_{5}$, that we present below together with the $\mathrm{NbSbCl}_{10}$ complex; and (ii) complexes based on $\mathrm{NbCl}_{5}$ that are relevant to liquid mixtures with other chlorides.

\subsection{The $\mathrm{Nb}_{2} \mathrm{Cl}_{10}, \mathrm{Nb}_{2} \mathrm{Br}_{10}$ and $\mathrm{NbSbCl} l_{10}$ Molecular Clusters}

As expected, all these clusters are formed by edge sharing of two distorted octahedra. The bond lengths between the metal ions and the terminal chlorines are almost unchanged relative to the isolated monomers, whereas those involving the two bonding halogens are considerably elongated (for instance, from $2.28 \AA$ to $2.58 \AA$ in $\mathrm{Nb}_{2} \mathrm{Cl}_{10}$ ). The bridging metal-halogen bonds are therefore weaker, and two pairs of axial bonds are slightly bent towards the ring forming the central part of the molecule (for instance, each of these bonds makes an angle of $86.6^{\circ}$ with the plane of the central ring in $\mathrm{Nb}_{2} \mathrm{Cl}_{10}$ ). Stretching of two bonds and bending of two other bonds are the main distortions of the basic octahedral unit upon edge sharing with another such unit.

The calculated binding energy of the two dimers relative to their isolated monomers is about $0.1 \mathrm{eV}$
Table 3. Frequencies of higher complexes based on $\mathrm{NbCl}$ (in $\mathrm{cm}^{-1}$ ) as calculated from the ionic model on molecular clusters (IM) and as obtained from vibrational spectroscopy of melts (M).

\begin{tabular}{lcccc}
\hline $\mathrm{NbCl}_{5}$ & IM & 394 & 314 & 153 \\
& M & 398 & 315 & 150 \\
$\mathrm{Nb}_{2} \mathrm{Cl}_{10}$ & IM & 415 & 255 & 165 \\
& M & 418 & 266 & 175 \\
$\mathrm{CsNbCl}_{6}$ & IM & 344 & 262 & 156 \\
& M & 373 & 281 & 175 \\
\hline
\end{tabular}

for $\mathrm{Nb}_{2} \mathrm{Cl}_{10}$ and about $0.2 \mathrm{eV}$ for $\mathrm{Nb}_{2} \mathrm{Br}_{10}$. The difference between these two cases arises from the higher polarizability of the bonding bromines. The calculated magnitude of the binding energy of the dimer accounts for the fact that, whereas the observed crystal structure is formed from dimers, a state of thermal equilibrium between monomers and dimers is established at the elevated temperatures of the melt. On the other hand, the calculated binding energy of $\mathrm{NbSbCl}_{10}$ relative to the isolated monomeric species is essentially zero. We find no evidence, therefore, in favour of the suggestion [9] that this complex may be stably bound in liquid mixtures of $\mathrm{NbCl}_{5}$ and $\mathrm{SbCl}_{5}$.

The top rows in Table 3 compare our results for the frequencies of three vibrational modes in $\mathrm{NbCl}_{5}$ and in its dimer with those reported by Brooker and Papatheodorou [9] from vibrational spectroscopic studies of melts. The agreement of our results with the data is quite pleasing.

\subsection{Complexes in Mixed Molten Salts: the $\mathrm{CsNbCl}_{6}$ Cluster}

Addition of an alkali chloride such as $\mathrm{CsCl}$ to molten $\mathrm{NbCl}_{5}$ yields by chlorine donation negatively charged $\mathrm{NbCl}_{6}$ octahedra, which are locally screened by alkali counterions. We have examined this process for the $\mathrm{NbCl}_{5}-\mathrm{CsCl}$ mixture at stoichiometry by evaluating the isolated $\mathrm{CsNbCl}_{6}$ molecular cluster. The main issue is how the Cs ion ties up to the $\mathrm{NbCl}_{6}$ octahedron. As is the case for the binding of an alkali ion to the tetrahedron formed by chlorination of a rare-earth chloride [14], the mechanically stable configurations are formed by placing the Cs counterion in either a twofold or a threefold coordination state. We find that the latter configuration, in which the Cs is bound to a face of the $\mathrm{NbCl}_{6}$ octahedron, is energetically more stable by about $0.3 \mathrm{eV}$. The octahedron is slightly distorted: the $\mathrm{Nb}$ ion has three terminal chlo- 
rines at $2.34 \AA$ and three bonding chlorines at $2.38 \AA$. The distance of the Cs ion from the latter is $3.8 \AA$.

A comparison between calculated vibrational frequencies in the $\mathrm{CsNbCl}_{6}$ cluster and data for the $\mathrm{NbCl}_{6}$ octahedron in molten halide mixtures [9] is reported in Table 3 .

\subsection{Complexes in Mixed Molten Salts: the $\mathrm{ANbCl}_{8}$ and $\mathrm{A}_{2} \mathrm{NbCl}_{11}$ Clusters with $\mathrm{A}=\mathrm{Al}$ or $\mathrm{Ga}$}

A fourfold, tetrahedral-like coordination of a group-IIIA metal ion such as $\mathrm{Al}$ or $\mathrm{Ga}$ by chlorines is strongly stable in molten trichlorides and in their mixtures with alkali chlorides. The fourfold coordination state is achieved in these pure trichloride melts by formation of $\mathrm{Al}_{2} \mathrm{Cl}_{6}$ or $\mathrm{Ga}_{2} \mathrm{Cl}_{6}$ dimers having the shape of edge-sharing distorted tetrahedra. The main issue in regard to the structure of liquid mixtures of these trichlorides with a pentachloride is whether (i) these dimeric units are preserved, or (ii) after dimer breakage the fourfold coordination of the trivalent element is preserved by chlorine sharing of tetrahedral-like units with pentachloride clusters.

We have examined the above issue by considering the $\mathrm{ANbCl}_{8}$ and $\mathrm{A}_{2} \mathrm{NbCl}_{11}$ isolated clusters corresponding to two different stoichiometries of the mixture, with $\mathrm{A}=\mathrm{Al}$ or $\mathrm{Ga}$. Considering first the case of $\mathrm{Al}$, for $\mathrm{AlNbCl}_{8}$ we have found two mechanically stable structures: a structure formed by corner sharing between an $\mathrm{NbCl}_{5}$ cluster and a distorted $\mathrm{AlCl}_{4}$ tetrahedron, and a second structure formed by edge sharing between an $\mathrm{NbCl}_{6}$ distorted octahedron and a distorted $\mathrm{AlCl}_{4}$ tetrahedron. The former structure is energetically more stable by about $0.3 \mathrm{eV}$. In the case of $\mathrm{Al}_{2} \mathrm{NbCl}_{11}$ we have found a mechanically stable structure consisting of an $\mathrm{NbCl}_{5}$ cluster and two distorted $\mathrm{AlCl}_{4}$ tetrahedra attached to it by corner sharing. All these structures are quite strongly bound against breakage into separate $\mathrm{NbCl}_{5}$ and $\mathrm{Al}_{2} \mathrm{Cl}_{6}$ units. Very similar results are obtained in the case $\mathrm{A}=\mathrm{Ga}$.

It thus appears from our calculations that the $\mathrm{NbCl}_{5}$ clusters in liquid mixtures with group-IIIA chlorides

[1] Z. Akdeniz, D. L. Price, M.-L. Saboungi, and M. P. Tosi, Plasmas and Ions 1, 3 (1998).

[2] M.-L. Saboungi, M. A. Howe, and D. L. Price, Molec. Phys. 79, 847 (1993).

[3] R. Triolo and A. H. Narten, J. Chem. Phys. 69, 3159 (1978). may act as efficient centres of aggregation for monomeric tetrahedral-like units. The evidence from vibrational spectroscopic studies of mixed melts has been presented in the review by Brooker and Papatheodorou [9]. They report that the vibrational spectra consist of overlapping bands that they attribute to $\mathrm{NbCl}_{5}, \mathrm{Nb}_{2} \mathrm{Cl}_{10}$, and $\mathrm{Al}_{2} \mathrm{Cl}_{6}$ molecules, but in the case of $\mathrm{NbCl}_{5}-\mathrm{GaCl}_{3}$ mixtures they suggest the possible formation of $\mathrm{GaNbCl}_{8}$ clusters.

\section{Summary}

In summary, we have proposed a model for the ionic interactions in the chlorides and bromides of some pentavalent metal elements from an analysis of their gaseous monomers and applied it to evaluate the structure and the cohesive and vibrational properties of higher molecular clusters. Our results confirm that a state of thermal equilibrium between molecular monomers and dimers can exist in the vapour and liquid phases of a compound such as Niobium pentachloride. We have also exposed two qualitatively distinct behaviours for ionic mixtures involving this compound: (i) chlorine donation through mixing with alkali halides stabilizes a sixfold octahedral-like coordination of the pentavalent element; and (ii) mixing with strongly bound units such as the tetrahedral clusters in chlorides of group-IIIA elements can preserve these units together with the fivefold or sixfold coordination of the pentavalent element through formation of higher complexes via corner or edge sharing. It is also hoped that these results on interionic force laws may be useful in computer simulation studies of these compounds in the molten state.

\section{Acknowledgements}

ZA acknowledges support from the Research Fund of the University of Istanbul under Project Number 1395 / 05052000. ZCÖ and ZA thank the Turkish Scientific and Technological Research Council (TUBITAK) for support.

[4] K. F. Ludwig, W. K. Warburton, L. Wilson, and A. I. Bienenstock, J. Chem. Phys. 67, 604 and 613 (1987).

[5] G. M. Photiadis and G. N. Papatheodorou, J. Chem. Soc. Dalton Trans. 1998, 981 (1998).

[6] Z. Akdeniz, A. Karaman, and M. P. Tosi, Z. Naturforsch. 56a, 376 (2001). 
[7] J. S. Ogden, W. Levason, E. G. Hope, J. T. Graham, D. M. Jenkins, and R. M. Angell, J. Molec. Struct. 222, 109 (1990).

[8] W. Hönle, S. Furuseth, and H. G. Schnering, Z. Naturforsch. 45b, 952 (1990).

[9] M. H. Brooker and G. N. Papatheodorou, in Advances in Molten Salt Chemistry, Vol. 5, ed. G. Mamantov, Elsevier, Amsterdam 1983, p. 26.

[10] M. Hargittai, Chem. Rev. 100, 2233 (2000).
[11] Z. Akdeniz and M. P. Tosi, Z. Naturforsch. 54a, 180 (1999).

[12] V. P. Spiridonov and G. V. Romanov, Bull. Moscow Univ. Ser. II 21, 526 (1966) and 22, 86 (1967).

[13] S. K. Kang, H. Tang, and T. A. Albright, J. Amer. Chem. Soc. 115, 1971 (1993).

[14] Z. Akdeniz, Z. Çiçek Önem, and M. P. Tosi, Z. Naturforsch. 56a, 721 (2001). 